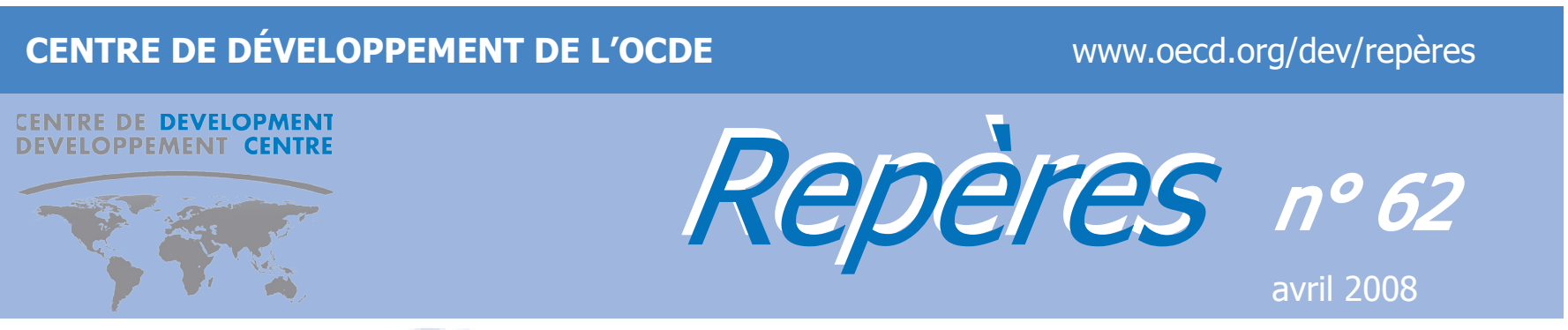

\title{
Investir dans la jeunesse africaine
}

\section{(Rédigé à partir du rapport Perspectives économiques africaines 2008*)}

par Lucia Wegner

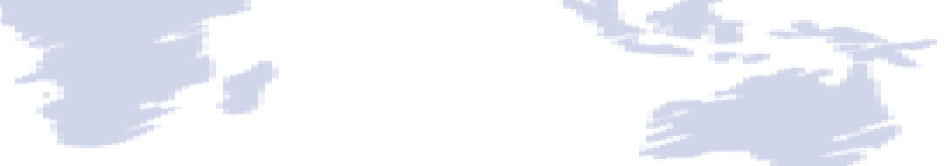

- Les pays africains souffrent d'un fort taux de chômage des jeunes et d'une pénurie de main-d'œuvre qualifiée.

- Les systèmes de formations techniques et professionnelles sont mal financés et mal gérés.

- Les stratégies de développement des compétences professionnelles doivent être intégrées dans les stratégies de réduction de la pauvreté

- Les ressources doivent se concentrer sur les secteurs dont les perspectives en termes d'emploi sont prometteuses.

Environ 133 millions de jeunes (la moitié de la jeunesse africaine) sont analphabètes. De nombreux jeunes n'ont aucune ou très peu de compétences professionnelles. Plus de 20 pour cent des jeunes de l'Afrique subsaharienne sont sans emploi. Ces chiffres parlent d'euxmêmes : la jeunesse africaine a besoin de formation professionnelle.

De meilleures compétences techniques sont essentielles pour améliorer la compétitivité et contribuer à l'inclusion sociale, la croissance de l'emploi et la réduction de la pauvreté. Le rapport Perspectives économiques africaines de 2008 passe en revue 35 pays et montre que les systèmes d'enseignement et de formation techniques et professionnels en Afrique souffrent d'un manque de personnel qualifié, d'équipements obsolètes, de programmes mal adaptés et déconnectés du marché du travail. Très peu de pays encouragent le développement des compétences dans le secteur informel, qui est pourtant le plus grand employeur et la principale source de formation en Afrique ${ }^{1}$.

Les compétences peuvent s'acquérir au sein d'institutions structurées et spécialisées, directement par immersion dans le monde du travail, ou par les deux biais - par une « double » formation. L'apprentissage traditionnel du secteur informel est prédominant : moins de 5 pour cent

1. Le secteur informel emploie 95 pour cent des travailleurs au Bénin, 90 pour cent au Cameroun, en Éthiopie et au Sénégal et 31 pour cent en Afrique du Sud. des apprentis passent par le système formel. Ainsi, au Sénégal, environ 400000 jeunes par an suivent un apprentissage, contre 7000 diplômés des centres de formation professionnelle formels ; au Ghana, plus de 80 pour cent du développement des compétences passe par le système de l'apprentissage.

En Afrique, les systèmes de formations techniques et professionnelles souffrent tant d'un manque de ressources (avec seulement 2 à 6 pour cent des budgets d'éducation), que d'un manque d'intérêt des instances internationales. Les donneurs n'ont été que des alliés incertains des programmes de formation. Lors de la conférence Éducation pour Tous à Jomtien en 1990 et du Sommet du Millénaire en 2000, l'éducation primaire a été mis en priorité. Cela a entraîné une baisse d'intérêt pour la formation professionnelle. Les écoles secondaires et les écoles offrant des formations professionnelles au Mozambique et en Afrique du Sud, par exemple, ont du mal à absorber les jeunes qui sortent de l'école primaire.

Cette situation de plus en plus difficile a remis la formation professionnelle au programme international, et de nombreux pays africains ont engagé la réforme de leur système de formation. Ils tendent à être plus performants lorsque l'objectif final est clairement perçu. Plus particulièrement, pour que les stratégies de formation aient un effet maximal, il est nécessaire qu'elles soient intégrées dans des plans de réduction de la pauvreté et qu'elles soient concentrées sur les secteurs dont les perspectives professionnelles sont les plus prometteuses. 
Les programmes doivent être flexibles et doivent répondre aux demandes du marché du travail, comme en Éthiopie, en Afrique du Sud et au Mozambique, où les manques spécifiques de compétences sont ciblés. Ils doivent être encadrés par des organismes indépendents comme le Technical Education, Vocational and Entrepreneurship Training Authority en Zambie, ou le Botswana Training Authority. Leur pertinence et leur impact sur le marché du travail doivent également être contrôlés et évalués.

Les partenariats avec les entreprises, les syndicats et les ONG peuvent aider à améliorer la pertinence des formations par rapport aux demandes du marché du travail. Les centres de formation, en Éthiopie par exemple, encouragent le secteur privé à participer à leur gestion, ce qui peut par ailleurs encourager les sources de financement privées. Au Maroc et en Éthiopie, des incitations financières encouragent les entreprises à favoriser la formation interne.

Il est important de prendre en considération le secteur informel. L'apprentissage traditionnel de compétences qui a lieu dans le secteur informel devrait être inclus dans les réformes des systèmes de formation techniques et professionnelles, comme c'est le cas pour les maîtres artisans en Ouganda, au Niger et au Mali. La certification des compétences acquises par l'apprentissage traditionnel, à l'exemple du certificat de compétence professionnelle au Bénin (Vocational Skill Certificate) établit un lien concret avec les besoins du secteur informel.
Afin d'évaluer les résultats de ce type de mesures, les observatoires du marché du travail au Bénin et au Rwanda suivent non seulement les changements dans le marché du travail mais aussi les carrières des diplômés des formations pour voir s'ils trouvent ou non un emploi. La généralisation de ce type d'observatoire à tous les pays africains représenterait un développement positif et significatif.

Les plans pour l'emploi peuvent servir de soutien ou de compléments à la formation. Le projet Femmes Africaines Chefs d'Entreprise (FACE) de la banque africaine de développement (BAD) et le Programme de développement des entreprises à fort potentiel de croissance appartenant à des femmes (GOWE) de I'OIT/BAD, deux plans plus spécifiquement destinés à l'accès des femmes au monde de l'entreprise, au Cameroun, en Éthiopie, au Kenya, en Tanzanie et en Ouganda sont des exemples à suivre.

Les pays africains diversifient leurs sources de financement du développement des compétences et la compétition entre les centres de formation publics et privés augmente. L'État maintient toutefois son rôle de fournisseur de formation pour les populations pauvres, défavorisées et vulnérables. Le défi est de développer et d'intégrer un cadre de financement qui puisse garantir des contributions équitablement réparties entre le gouvernement, les communautés locales, l'industrie et les jeunes en formation.

Le potentiel d'employabilité de la jeunesse africaine, et leur futur, en dépend.

* Il s'agit d'un rapport conjoint de la Banque africaine de développementet du Centre de développement de I'OCDE, avec la Commission Économique pour l'Afrique des Nations unies, qui a bénéficié du soutien financier de la Commission européenne.

Les lecteurs sont invités à citer ou reproduire les informations des Repères du Centre de développement de I'OCDE dans leurs propres publications. En échange, le Centre demande les remerciements de rigueur ainsi qu'un exemplaire de la publication. Le texte intégral des Repères et d'autres informations sur le Centre de développement et ses travaux sont disponibles sur : www.oecd.org/dev
Centre de développement de I'OCDE 2, rue André-Pascal, 75775 Paris Cedex 16, France Tél : $33(0) 145.24 .82 .00$

Fax : $33(0) 144.30 .61 .49$ mél : cendev.contact@oecd.org 